\title{
Resistivity Imaging of Mataloko Geothermal Field By Mise-Á-La-Masse Method
}

\author{
Enjang Jaenal Mustopa ${ }^{1)}$, Edi Suhanto ${ }^{2)}$, Wahyu Srigutomo ${ }^{1)}$ and Doddy Sutarno ${ }^{1)}$ \\ 1) Physics of Complex System Division, \\ Faculty of Mathematics and Natural Sciences, \\ Bandung Institute of Technology. \\ 2) Geothermal Research Group, Geological Resources Center \\ e-mail:enjang@fi.itb.ac.id
}

Received 14 October 2010, Revised 11 December 2010, Accepted 27 January 2011

\begin{abstract}
Mise-á-la-masse (MAM) measurement has been carried out in the Mataloko geothermal field, Flores, East Nusa Tenggara. The survey was conducted along 16 survey lines in a radial configuration to collect 176 sounding sites. The purpose of the measurement is to image a promising reservoir zone and to correlate the results with the previous geosciences surveys for locating the best drilling target. Three-dimensional (3D) inversion scheme was applied to interpret MAM data. The subsurface resistivity structure obtained from $3 D$ inversion result shows that a low resistivity (1 - 10 ohm.m) structure is widely distributed in the southwest and northeast of the well MT-3/MT-5 at the depth $0-100 \mathrm{~m}$. On the other hand, in the deeper part of the survey area, it is recognized that a resistivity in the western part is lower than in the eastern part of the area. The fluid flow tests of well MT-5 produced dry saturated steams of 19.3, 12.6 and $17.4 \mathrm{ton} / \mathrm{h}$ at wellhead pressures of 4.0, 6.0, and $5.0 \mathrm{kscg}$, respectively. Furthermore, the fluid has high enthalpies whose values are 2746, 2755, 2763, 2768, and $2771 \mathrm{~kJ} / \mathrm{kg}$ at wellhead pressure of 4.0, 5.0, 6.0, 7.0, and $7.5 \mathrm{kscg}$, respectively. The incorporation of MAM data analyses results with the geosciences data makes it possible to recommend the location of the best target for the drilling site.
\end{abstract}

Keywords: Mise-á-la-masse method, 3D inversion, Mataloko geothermal field

\section{Introduction}

The mise-á-la-masse (MAM) method has been used for years since its first introduction by Schlumberger in 1920 as reported by Parasnis ${ }^{1)}$ in geophysical explorations for mapping the extent buried conductive body. The method utilizes a pair of current electrodes where the positive current electrode is directly planted in the mineralized or conductive body through a borehole, whereas the negative current electrode is placed on the surface at a large distance away that can be regarded as infinity. If the conductivity of the anomalous body is very high, there is a relatively little potential drop across the body itself and the buried body can be mapped on the surface of the earth or in boreholes as a zone of anomalous potential ${ }^{2)}$.

The MAM method has been used for prospecting vine-type mineralized bodies where the measured potential field reflects the shape, size, and orientation of the mineral deposit within limestone and mafic gneiss ${ }^{3)}$; surveying an auriferous deposit of sulfide $^{4)}$, determination of coal barrier thickness and continuity as well as the structural and depositional pinchouts in a coal mine ${ }^{5)}$. It can also be applied in delineation of contaminant plume in groundwater that migrates via lateral and vertical pathways within stratigraphic units ${ }^{6)}$; and in detection of water or mud in fractured vadose zone karst environtment ${ }^{7}$. Interpretation of potential fractures in geothermal system that is based on MAM data has been applied to monitor fluid flow behaviors during water injection and steam production operations ${ }^{8)}$.

In a geothermal exploration, the MAM method has primarily been executed using a well casing as the buried electrode in an existing hole. The configuration of MAM survey is presented in the Figure 1 . The electrode $C_{1}$ connected to the casing pipe of a well is earthed into the deep formation under the investigated anomalous body, on the other hand, the electrode $C_{2}$ is located at a great distance from the borehole on the surface. The fixed potential electrode $P_{2}$ is also set very far from the borehole, usually on the opposite side of $C 2$. The potential electrode $P_{1}$ is moved radially forming concentric points with an appropriate separation between each of them from the target (borehole). A commutated electric current is injected into the earth and the resulting potentials on the ground surface that are measured at stations (various location of $P_{1}$ ) by mean of a voltmeter with respect to the base station $P_{2}$.

The MAM survey has been carried out in the Mataloko geothermal field, Flores, East Nusa Tenggara (Figure 2). The survey was conducted along 16 survey lines in a radial configuration to collect 176 sounding sites. The purpose of the measurement is to delineate a subsurface resistivity structure correlating to the existing of geothermal reservoir in the survey area.

The residual potential, apparent resistivity mapping and 3D inversion analyses of MAM data are presented in this paper. The $3 \mathrm{D}$ inversion of MAM 
data employed here is a singularity removal algorithm based on the least squares deconvolution of the apparent resistivity ${ }^{9}$. The MAM analyses results will be incorporated with the previous geosciences surveys to image a promising reservoir zone for locating the best drilling target.

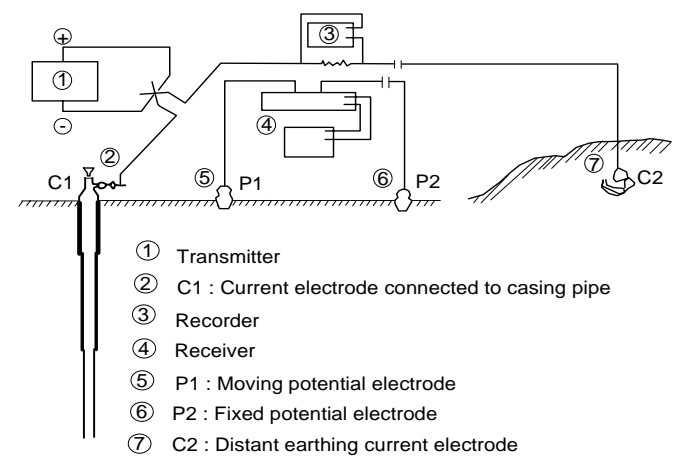

Figure 1. Configuration of MAM survey

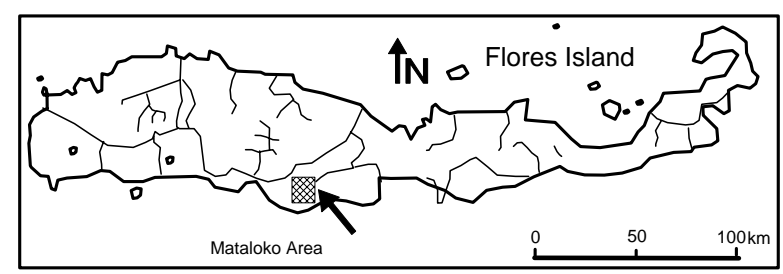

Figure 2. Location Mataloko area

\section{Theoretical background}

\subsection{Resistivity maping}

If the potential at an infinite distance is assumed to be zero, the potential due to a point source of a current electrode at a distance $r$ on the ground surface of an isotropic and homogenous earth is

$$
V_{r}=\frac{\rho I}{2 \pi} \frac{1}{r} .
$$

The potential at $\mathrm{P} 1$ due to point source $C_{1}$ can be derived by a process of integration starting from the potential of a point source of current, which is

$$
V_{P_{1} C_{1}}=\frac{\rho I}{2 \pi} \frac{1}{l} \ln \left(\frac{l+\sqrt{l^{2}+{\overline{P_{1} C_{1}}}^{2}}}{\overline{P_{1} C_{1}}}\right),
$$

and the potential due to point source $C_{2}$ is

$$
V_{P_{1} C_{2}}=\frac{\rho I}{2 \pi}\left(\frac{1}{\overline{P_{1} C_{2}}}\right),
$$

where $\rho$ is resistivity of half space, $l$ is a length of casing pipe, $\overline{P_{1} C_{1}}$ and $\overline{P_{1} C_{2}}$ are the distance $P_{1}-C_{1}$ and $P_{1}-C_{2}$, respectively. The total potential at $P_{1}$ due to point source $C_{1}$ and $C_{2}$ is

$$
\begin{aligned}
V_{P_{1}} & =V_{P_{1} C_{1}}+V_{P_{1} C_{2}} \\
& =\frac{\rho I}{2 \pi}\left\{\frac{1}{l} \ln \left(\frac{l+\sqrt{l^{2}+{\overline{P_{1} C_{1}}}^{2}}}{\overline{P_{1} C_{1}}}\right)-\frac{1}{\overline{P_{1} C_{2}}}\right\} .
\end{aligned}
$$

Doing a similar way with the operation above by using $P_{2}$ instead of $P_{1}$, the total potential at $P_{2}$ due to point source $C_{1}$ and $C_{2}$ is

$$
\begin{aligned}
V_{P_{2}} & =V_{P_{2} C_{1}}+V_{P_{2} C_{2}} \\
& =\frac{\rho I}{2 \pi}\left\{\frac{1}{l} \ln \left(\frac{l+\sqrt{l^{2}+{\overline{P_{2} C_{1}}}^{2}}}{\overline{P_{2} C_{1}}}\right)-\frac{1}{\overline{P_{2} C_{2}}}\right\} .
\end{aligned}
$$

The potential different between $P_{1}$ and $P_{2}$ is

$$
\begin{aligned}
& V_{P_{1} P_{2}}=V_{P_{1}}-V_{P_{2}}
\end{aligned}
$$

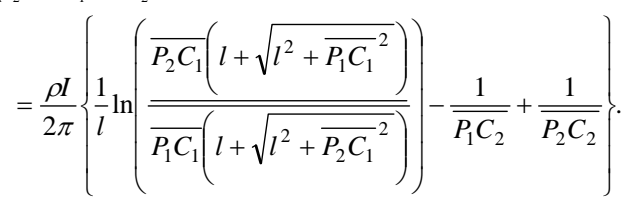

Therefore, the expression for the apparent resistivity for the four-electrode configuration used in the field survey is expressed as

$$
\rho_{a}=G \frac{V}{I},
$$

where $G$ is geometrical factor,

$$
G=2 \pi\left\{\frac{1}{l} \ln \left(\frac{\overline{P_{2} C_{1}}\left(l+\sqrt{l^{2}+\overline{P_{1} C_{1}^{2}}}\right)}{\overline{P_{1} C_{1}\left(l+\sqrt{l^{2}+\overline{P_{2} C_{1}^{2}}}\right)}}-\frac{1}{\overline{P_{1} C_{2}}}+\frac{1}{\overline{P_{2} C_{2}}}\right\}^{-1} .\right.
$$

For the directional well casing, the potential at point $P(x, y)$ on the ground surface as shown in the Figure 3 can be derived by a process of integration starting from $O(x, y, z)$ to an arbitrary length of the casing pipe, that is 


$$
\begin{aligned}
& V(P)=\frac{\rho I}{2 \pi\left(l_{1}+l_{2}\right)}\left\{\ln \left(\frac{l_{1}+\sqrt{l_{1}^{2}+r^{2}}}{r}\right)+\cos \theta .\right. \\
& \left.\ln \left(\frac{l_{1} \cos \theta+l_{2}-r \sin \theta \cos (\omega-\phi)+\sqrt{r^{2}+l_{1}{ }^{2}+l_{2}{ }^{2}+2 l_{1} l_{2} \cos \theta-2 r l_{2} \sin \theta \cos (\omega-\phi)}}{l_{1} \cos \theta-r \sin \theta \cos (\omega-\phi)+\sqrt{r^{2}+l_{1}{ }^{2}}}\right)\right\} .
\end{aligned}
$$

The expression for the apparent resistivity for the general configuration is the same as with the Equation (3). However, the geometrical factor $G$ for a directional well can be derived as follows:

$$
\begin{aligned}
& G=2 \pi\left(l_{1}+l_{2}\right)\left\{\ln \left(\frac{l_{1}+\sqrt{l_{1}^{2}+r^{2}}}{r}\right)+\cos \theta \mathrm{X}\right. \\
& \left.\ln \left(\frac{l_{1} \cos \theta+l_{2}-r \sin \theta \cos (\omega-\phi)+\sqrt{r^{2}+l_{1}^{2}+l_{2}^{2}+2 l_{1} l_{2} \cos \theta-2 r l_{2} \sin \theta \cos (\omega-\phi)}}{l_{1} \cos \theta-r \sin \theta \cos (\omega-\phi)+\sqrt{r^{2}+l_{1}^{2}}}\right)\right\}^{-1} .
\end{aligned}
$$

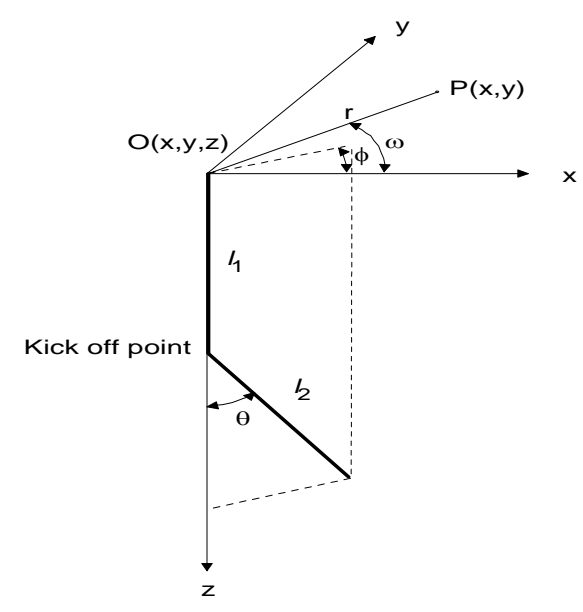

Figure 3. Electric potential for a directional casing pipe $^{10)}$

\subsection{Three Dimensional Inversion}

The 3D inversion procedure of MAM data used in the paper is least squares deconvolution technique ${ }^{9,11)}$. The resistivity inverse problem can be expressed as

$$
\Delta d=A \Delta p,
$$

where $\Delta d$ is the vector of differences between modeled responses and data, $\Delta p$ is the correction vector to the initial model parameters $p_{o}$ and $A$ is a Jacobian matrix. A common approach for the model parameterization is to divide the model into blocks of unknown constant resistivity. To scale both the parameters and the data so that the Jacobian will have a stable inverse, the logarithms of model resistivity and measured apparent resistivity are used. Because the resistivity problem is generally ill-posed, it is necessary to use some constraints on $\Delta p$ to insure the stability of the iterative process, that is, smoothness constrain to a least-squares formulation.

The objective function to be minimize is ${ }^{10)}$

$$
U=\|\Delta d-A \Delta p\|^{2}+\lambda\|r\|^{2},
$$

where $r$ is a roughness matrix, $\lambda$ is Lagrange multiplier, and \|\| denotes the Euclidean norm. The minimization of Equation (10) produces the system of linear equations

$$
\left(A^{T} A+\lambda C^{T} C\right) \Delta p=A^{T} \Delta d,
$$

that can be solved by using the modified GramSchmidt method which solves the least square problem.

The important thing in the inversion procedure is to calculate sensitivity matrix, that is partial derivative of apparent resistivity with respect to model resistivities.

$$
\frac{\partial \phi}{\partial \rho}=\rho^{-2} \int_{V} \nabla \phi \nabla \phi^{\prime} d \tau
$$

For the block model shown in the Figure 4, the average of apparent resistivity within each block is

$$
\log \rho_{m}=\frac{1}{M} \sum_{i=1}^{M} \log \rho_{a i} .
$$

The potential due to the current source and potential electrode are $^{12)}$ 


$$
\begin{aligned}
\phi= & \frac{\rho I_{l}}{2 \pi}\left\{\frac{1}{\left|\vec{r}_{c}\right|}+\frac{1}{\left|\vec{r}_{c}^{*}\right|}\right\} \\
= & \frac{\rho I_{l}}{2 \pi}\left\{\frac{1}{\sqrt{\left(x-x_{c}\right)^{2}+\left(y-y_{c}\right)^{2}+\left(z-z_{c}\right)^{2}}}+\right. \\
\phi^{\prime} & \left.=\frac{\rho}{2 \pi} \frac{1}{\sqrt{\left(x-x_{c}\right)^{2}+\left(y-y_{c}\right)^{2}+\left(z+z_{c}\right)^{2}}}\right\}, \\
& =\frac{\rho}{2 \pi} \frac{1}{\sqrt{\left(x-x_{c}\right)^{2}+\left(y-y_{c}\right)^{2}+z^{2}}}
\end{aligned}
$$

The Equation (14) becomes

$$
\frac{\partial \varphi}{\partial \rho}=\frac{I_{l}}{4 \pi^{2}} \int_{V}\left\{\frac{\vec{r}_{c} \cdot \vec{r}_{p}}{\left|\vec{r}_{c}\right|^{3}\left|\vec{r}_{p}\right|^{3}}+\frac{\vec{r}_{c}^{*} \cdot \vec{r}_{p}}{\left|\vec{r}_{c}^{*}\right|^{3}\left|\vec{r}_{p}\right|^{3}}\right\} d \tau
$$

For line source problem, the Equation (18) becomes

$$
\frac{\partial \varphi}{\partial \rho}=\frac{I_{l}}{4 \pi^{2}} \int_{0}^{l} \int_{V}\left\{\frac{\vec{r}_{c} \cdot \vec{r}_{p}}{\left|\vec{r}_{c}\right|^{3}\left|\vec{r}_{p}\right|^{3}}+\frac{\vec{r}_{c}^{*} \cdot \vec{r}_{p}}{\left|\vec{r}_{c}^{*}\right|^{3}\left|\vec{r}_{p}\right|^{3}}\right\} d \tau d z_{c},(
$$

where $l$ is a length of line source.

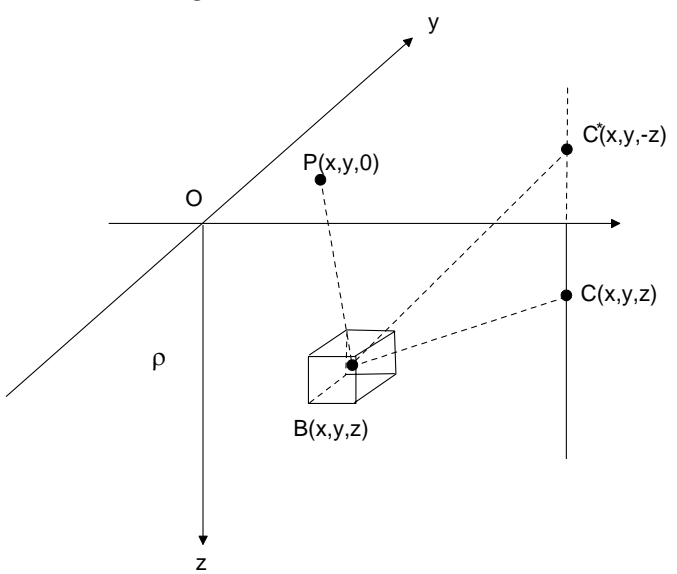

Figure 4. Three dimensional model of a line source model $^{13)}$

\section{MAM Field Survey}

The MAM survey has been conducted in Mataloko Geothermal Field, East Nusa Tenggara by utilizing the anchor casing of the wells of MT-3 and MT-5 as current electrodes $C_{1}$ (Figure 5). The purpose of the survey was to delineate the possible extension of geothermal reservoir in the area. The distance of electrode $C_{2}$ was located in the southwest of the surveyed area around $4 \mathrm{~km}$ away form the current electrode $C_{1}$. The potential electrode $P_{2}$ was set in the northeast of the surveyed area about $2.5 \mathrm{~km}$ away from the current electrode $C_{1}$ in the opposite direction of the current electrode $C_{2}$. The potential electrode $P_{1}$ was moved radially from the $C_{1}$ electrode with $100 \mathrm{~m}$ separation. The casings of MT-3 and MT-5 are vertical, which have a depth of $613 \mathrm{~m}$ and $378 \mathrm{~m}$, respectively. Therefore, we used a vertical well case for both current sources for calculating potential and apparent resistivity.

The MAM measurements were conducted along 16 survey lines in a radial configuration to the wells. Every line has 11 points of potential measurements. Therefore, the total measurements of potential difference in this survey were176 points for each current source.

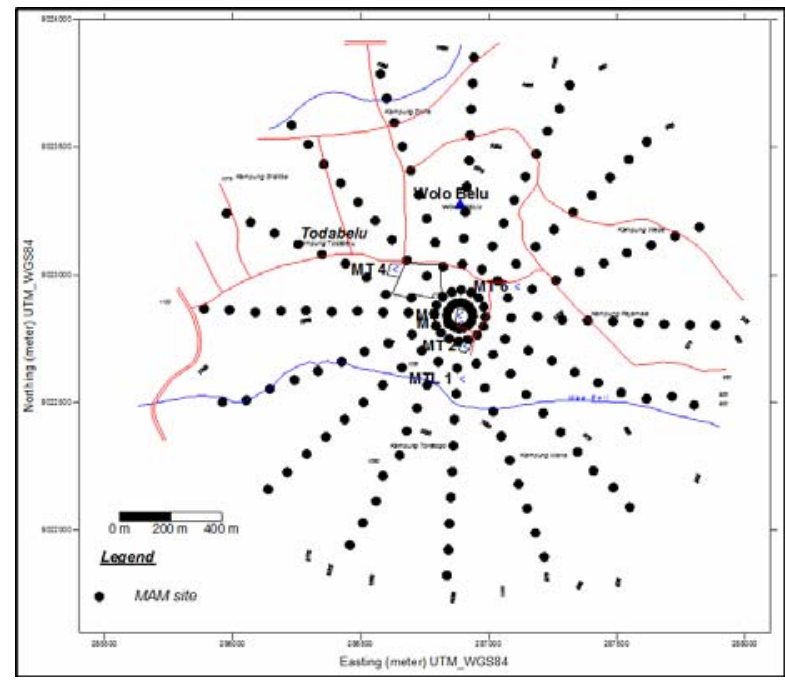

Figure 5. Location of MAM survey

\section{Field Data Analysis}

The actual potential difference $V$ measured at each station was transformed into apparent resistivity using Equation (7). The apparent resistivity value represents the total responses of the subsurface conditions. In order to recognize response of any subsurface anomalous body, the values of observed potential, residual potential and apparent resistivity are presented in this paper.

The interpretation of the MAM data can be analyzed directly from the processing results. Moreover, to get better understanding of the subsurface resistivity distribution in the Mataloko geothermal field, the 3D inversion technique of MAM data will be also presented in this paper.

\subsection{Potential distribution}

The observed potential distribution maps of well MT-3 is presented in the Figure 6. From the figure, it can be seen that the observed potential values $V / I$ of well MT-3 is ranging from 0.3 to 3.3 $\mathrm{mV} / \mathrm{A}$. The contour maps of $V / I$ show concentric circles at the center of measurement (current source electrode). 


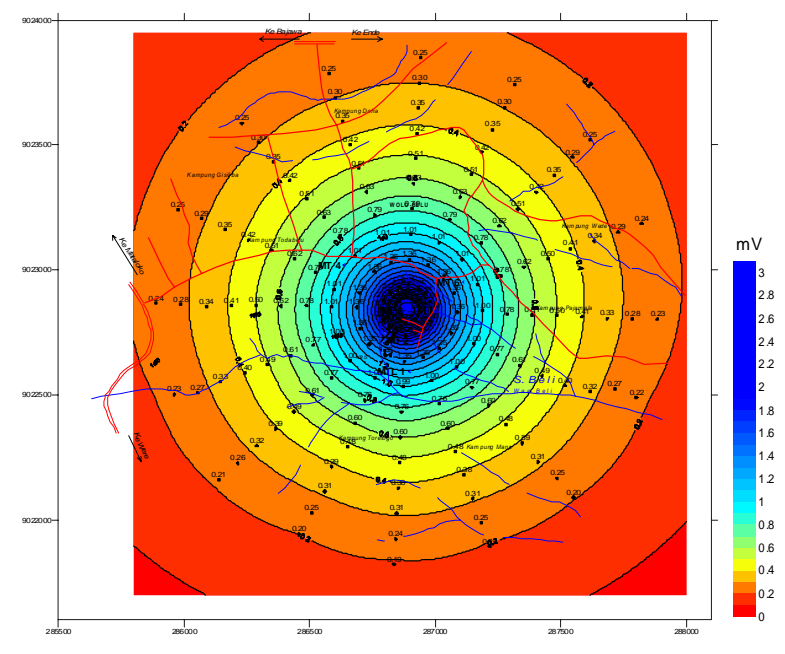

Figure 6. Observed potential of the well MT-3

\subsection{Residual potential distribution}

Figure 7 shows a residual potential map from a charged potential of well MT-3. Residual potential is calculated from the observed potential values subtracted by the theoretical potential values. It is recognized from the figure that the residue potential of well MT-3 is ranging from -1.2 to $0.3 \mathrm{mV} / \mathrm{A}$. A low (negative) residual potential anomaly is distributed at the center of measurement site and at the eastern of the well MT-3. On the contrary, the high residual potential (positive) is distributed widely at the western part of the area.

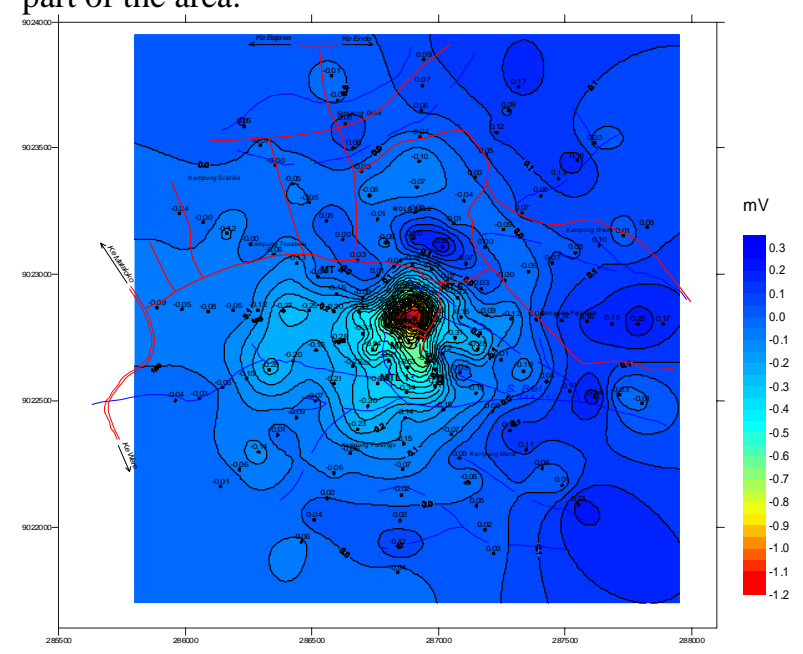

Figure 7. Residual potential map of the well MT-3.

\subsection{Apparent resisitivy distribution}

Figure 8 shows the contoured-map of the apparent resistivities obtained from the field survey with a current source electrode at well MT-3. The apparent resistivity values are ranging from 1 to 15 ohm-m. The low resistivity contours (less than 10 ohm-m) are distributed in the center and in the western-half of the surveyed area. On the contrary, the high resistivity (more than $10 \mathrm{ohm}-\mathrm{m}$ ) dominates the eastern part of the area.

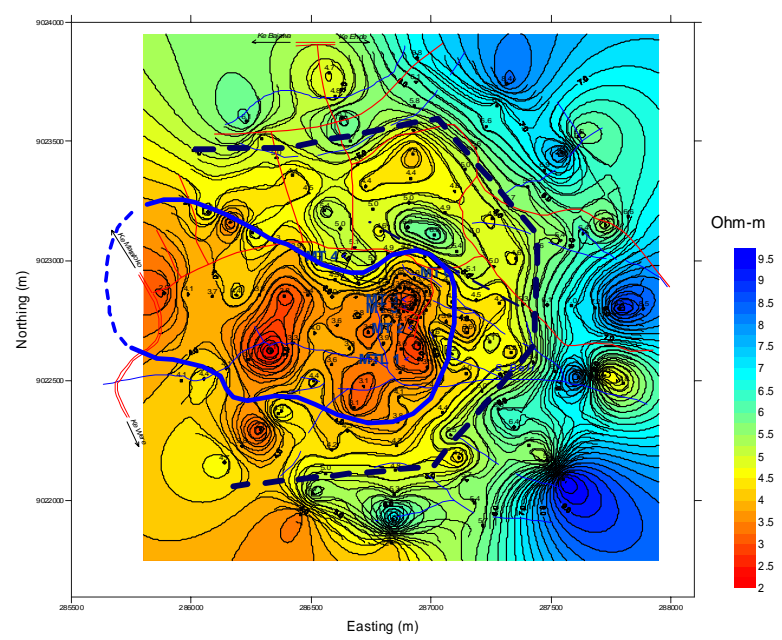

Figure 8. Apparent resistivity map of the well MT-3.

Generally the low resistivity zone is widely distributed from the center to the west. On the other hand, the high resistivity is widely distributed in the Southeast. Furthermore, there is extremely low resistivity zone indicated by bold line in the Figure 8 (the value of resistivity $<4$ ohm-m) covering manifestation wells of MT-2, MT-3, and MT-5.

\subsection{3-D inversion Analysis}

Three-dimensional inversion results of the MAM data using charged currents of MT-3 and MT-5 are displayed in the Figure 9. The smoothing factor used in the inversion procedure is 0.005 . The finitedifference mesh used in this inversion consists of $15 \mathrm{x}$ 15 x 7 (1575) nodes or 1176 blocks. While, the initial guesses of the resistivity of all blocks were set to 7 ohm-m (homogenous earth model), that is obtained from the average value of apparent resistivity for all measurements.

In the near surface of the surveyed area, a low resistivity (1-10 ohm-m) is widely distributed in the southwest and Northeast of the well MT-3/MT-5 at depths of $0-100 \mathrm{~m}$ as shown in the Figure 9. Another low resistivity zone is distributed in the east of the area. On the contrary, the high resistivity zone is concentrated in the Southeast. The resistivity distribution becomes higher and more intense in the deeper part except in the Southeast.

According to the subsurface resistivity structure in the deeper part shown in the Figure 9, it is clearly recognized that there is a boundary between the northwestern and southwestern part of the area, which is considered as a fault extending from southeast to northwest. The low resistivity distribution is shallower and thinner in the northwest than that in the southeast. 


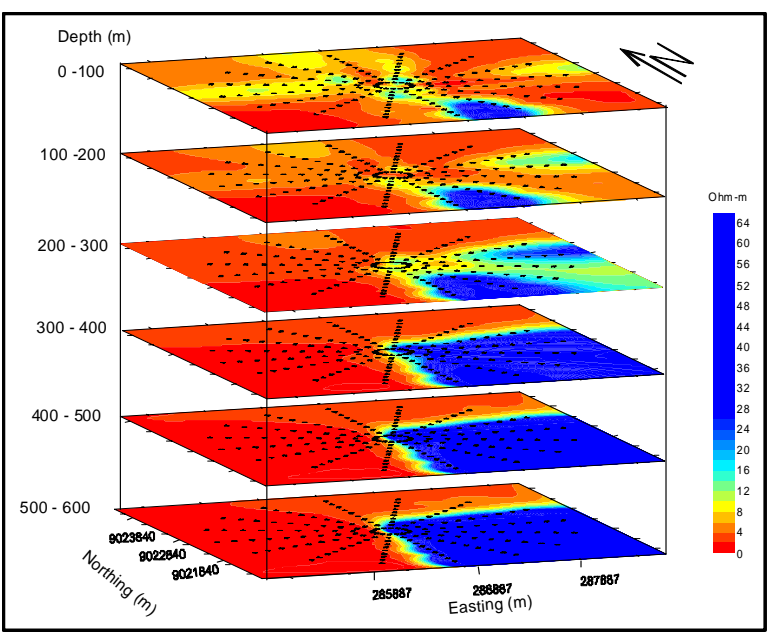

Figure 9. 3D inversion results

Wells MT-4 and MT-6 located in the boundary between the low resistivity zone and the high resistivity zone. The boundary area is a high-lost circulation zone while drilling of the MT-6, expected as the existing of a fracture zone extending from northwest to southeast, which is a requirement for a promising geothermal reservoir. It is confirmed from the drilling data that the MT-5 produced dry saturated steams of $19.3,12.6$ and $17.4 \mathrm{ton} / \mathrm{h}$ at wellhead pressures of $4.0,6.0$, and $5.0 \mathrm{kscg}$, respectively. Extrapolation data estimated a maximum flow of about 20 ton/h at wellhead pressure of $4.0 \mathrm{kscg}^{14)}$. The fluid has relatively stable high entalphies of 2746, 2755, 2763, 2768, and $2771 \mathrm{~kJ} / \mathrm{kg}$ at wellhead pressure of $4.0,5.0,6.0,7.0$, and $7.5 \mathrm{kscg}$, respectively, which may indicate the presence of a single steam supply system that feeds the well. The presence of isothermal profile (about $168{ }^{\circ} \mathrm{C}$ ) down to depth of $365 \mathrm{~m}^{14)}$ also supported the idea. Based on that consideration, the location of the best target for drilling site is in the west of the MT-3/M-5 of the low resistivity zone near the surface.

\section{Conclusions}

The MAM method performed in the Mataloko Geothermal Field has recognized an anomalously low resistivity zone which may correlate with the existing geothermal reservoir. Furthermore, the good correlation between MAM data interpretation results and the drilling data has been enabled to locate the promising wells.

According to the subsurface resistivity structure in the deeper part obtained from 3D inversion results, it is clearly recognized that there is a boundary between the southeastern and northwest part of the area, which is considered as a fault extending from Southeast to Northwest.

Generally the low resistivity zone is widely distributed from the center to the west. On the other hand, the high resistivity is widely distributed in the Southeast of the area. Furthermore, there is extremely low resistivity zone (the value of resistivity $<4$ ohm- m) covering manifestation wells of MT-2, MT-3, and MT-5.

Wells MT-4 and MT-6 are located in the boundary between the low resistivity zone and the high resistivity zone. The boundary area is a high-lost circulation zone while drilling of the MT-6, expected as the existing of a fracture zone extending from Northwest to Southeast, which is a requirement for a promising geothermal reservoir.

Based on the consideration of the MAM data interpretation results incorporated with the geosciences data, the location of the best target for drilling site is in the west of the well MT-3/M-5 of the low resistivity zone in the near surface.

\section{References}

1. D. S. Parasnis, Mining geophysics, Elsevier, Amsterdam, 195-199, 343-345, 1966.

2. K. Ushijima, Exploration of Geothermal Reservoir by the Mise-Á-La-Masse Measurement, Geothermal Resources Council Bulletin, February, 17-25, 1989.

3. L. Mansinha and C. J. Mwenifumbo, A mise-á-lamasse study at the Cavendish Geophysical Test Site, Geophysics, 48(9), 1252-1257, 1983.

4. B. B. Bhattacharya et al., Mise-á-la-masse Survey for an Auriferous Sulfide Deposit, Geophysics, 66(1), 70-77, 2001.

5. R. Rodriguez and H. R. Rodriguez, Applying the Mise-á-la-masse Method to Coal Mining Problems, The Leading Edge - SEG, July, 759762, 2000.

6. J. L. Osiensky and P. R. Donaldson, A Modified Mise-á-la-masse Method for Contaminant Plume Delineation, Ground Water, 32(3), 448-457, 1994.

7. J. E. Nyquist, H. E. Mintz, and M. J. S. Roth, Mise-á-la-masse and Smoke Test for Mapping Vadose Zone Karst, The Leading Edge - SEG, November, 1510-1515, 2008.

8. H. Hatanaka et al., Three-Dimensional Modeling and Inversion of the Mise-a-la-masse Data Using a Steel-Casing Borehole, Proc. World Geothermal Congress 2005, Antalya, Turkey, 24-29 April, 14, 2005.

9. H. Mizunaga et al., Three-dimensional Inversion of the mise-á-la-masse Data, Geothermal Research Society of Japan-Annual Meeting, Abstract-A1, 1996.

10. K. Ushijima et al., Vertical electric profiling in Kitanigoro Area, Proc. of the second SEGJ International Symposium on Geotomography, 2, 261-270, 1992.

11. M. H. Loke and R. D. Barker, R. D., Leastsquares Deconvolution of Apparent Resistivity Pseudosections, Geophysics, 60(6), 1682-1690, 1995. 
12. Y. Sasaki, Two-Dimensional Joint Inversion of Magnetotelluric and Dipole-Dipole Resistivity, Geophysics, 55, 682-694, 1989.

13. P. Sumintadireja, S. Sudarman, and K. Ushijima, Mise-á-la-masse and gravity data surveys at the Kamojang Geothermal Field, Proc. WGC 2000, Kyushu-Tohoku, 1777-1784, 2000.
14. Drilling exploration team of well MT-5 in Mataloko Geothermal Field, 2005. The report of drilling exploration of well MT-5 in Mataloko Geothermal Field Mataloko, Ngada District, East Nusatenggara (NTT), Geological Resources Centre, Bandung, Indonesia. 